UCRL-MA-147996

REV 2

\title{
SLURM: Simple Linux Utility for Resource Management
}

Moe Jette and Mark Grondona

December 19, 2002

U.S. Department of Energy

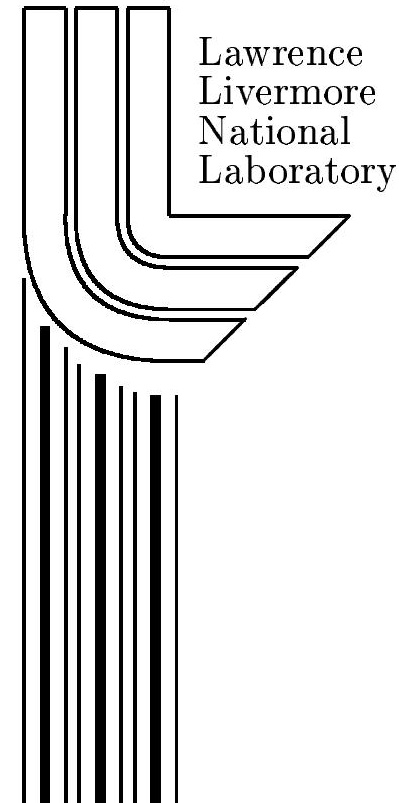




\section{DISCLAIMER}

This document was prepared as an account of work sponsored by an agency of the United States Government. Neither the United States Government nor the University of California nor any of their employees, makes any warranty, express or implied, or assumes any legal liability or responsibility for the accuracy, completeness, or usefulness of any information, apparatus, product, or process disclosed, or represents that its use would not infringe privately owned rights. Reference herein to any specific commercial product, process, or service by trade name, trademark, manufacturer, or otherwise, does not necessarily constitute or imply its endorsement, recommendation, or favoring by the United States Government or the University of California. The views and opinions of authors expressed herein do not necessarily state or reflect those of the United States Government or the University of California, and shall not be used for advertising or product endorsement purposes.

This work was performed under the auspices of the U. S. Department of Energy by the University of California, Lawrence Livermore National Laboratory under Contract No. W-7405-Eng-48.

This report has been reproduced directly from the best available copy.

Available electronically at http://www.doc.gov/bridge

Available for a processing fee to U.S. Department of Energy

And its contractors in paper from

U.S. Department of Energy

Office of Scientific and Technical Information

P.O. Box 62

Oak Ridge, TN 37831-0062

Telephone: (865) 576-8401

Facsimile: (865) 576-5728

E-mail: reports@adonis.osti.gov

Available for the sale to the public from

U.S. Department of Commerce

National Technical Information Service

5285 Port Royal Road

Springfield, VA 22161

Telephone: (800) 553-6847

Facsimile: (703) 605-6900

E-mail: orders@ntis.fedworld.gov

Online ordering: http://www.ntis.gov/ordering.htm

OR

Lawrence Livermore National Laboratory

Technical Information Department's Digital Library

http:/ / www.llnl.gov/tid/Library.html 


\title{
SLURM: Simple Linux Utility for Resource Management
}

\author{
Moe Jette \\ Mark Grondona \\ \{jette,mgrondona\}@llnl.gov
}

December 19, 2002

\begin{abstract}
Simple Linux Utility for Resource Management (SLURM) is an open source, fault-tolerant, and highly scalable cluster management and job scheduling system for Linux clusters of thousands of nodes. Components include machine status, partition management, job management, scheduling and stream copy modules. This paper presents a overview of the SLURM architecture and functionality.
\end{abstract}




\section{Overview}

SLURM $^{1}$ (Simple Linux Utility for Resource Management) is a resource management system suitable for use on Linux clusters, large and small. After surveying[7] resource managers available for Linux and finding none that were simple, highly scalable, and portable to different cluster architectures and interconnects, the authors set out to design a new system.

The result is a resource management system with the following general characteristics:

- Simplicity: SLURM is simple enough to allow motivated end users to understand its source code and add functionality. The authors will avoid the temptation to add features unless they are of general appeal.

- Open Source: SLURM is available to everyone and will remain free; its source code is distributed under the GNU General Public License.

- Portability: SLURM is written in the C language, with a GNU autoconf configuration engine. While initially written for Linux, other UNIX-like operating systems should be easy porting targets.

- Interconnect independence: SLURM supports UDP/IP based communication and the Quadrics Elan3 interconnect. Adding support for other interconnects, including topography constraints, is straightforward. Users select from the supported interconnects at compile time via GNU autoconf.

- Scalability: SLURM is designed for scalability to clusters of thousands of nodes. The SLURM controller for a cluster with 1000 nodes occupies on the order of $2 \mathrm{MB}$ of memory and performance is excellent.

- Fault tolerance: SLURM can handle a variety of failure modes without terminating workloads, including crashes of the node running the SLURM controller.

- Secure: SLURM employs crypto technology to authenticate users to services and services to each other. SLURM does not assume that its networks are physically secure, but does assume that the entire cluster is within a single administrative domain with a common user base across the entire cluster.

- System administrator friendly: SLURM is configured with a few simple configuration files and minimizes distributed state. Its interfaces are usable by scripts and its behavior is highly deterministic.

\footnotetext{
${ }^{1} \mathrm{~A}$ tip of the hat to Matt Groening and creators of Futurama, where Slurm is the highly addictive soda-like beverage made from worm excrement.
} 


\subsection{What is SLURM?}

As a cluster resource manager, SLURM has three key functions. First, it allocates exclusive and/or non-exclusive access to resources (compute nodes) to users for some duration of time so they can perform work. Second, it provides a framework for starting, executing, and monitoring work (normally a parallel job) on the set of allocated nodes. Finally, it arbitrates conflicting requests for resources by managing a queue of pending work.

Users interact with SLURM through four command line utilities: srun for submitting a job for execution and optionally controlling it interactively, scancel for early termination of a pending or running job, squeue for monitoring job queues, and sinfo for monitoring partition and overall system state. System administrators perform privileged operations through an additional command line utility: scontrol.

External schedulers and meta-batch systems can submit jobs to SLURM, order its queues, and monitor SLURM state through an application programming interface (API).

The central controller daemon, slurmctld maintains the global state and directs operations. Compute nodes simply run a slurmd daemon (similar to a remote shell daemon) to export control to SLURM.

\subsection{What SLURM is Not}

SLURM is not a sophisticated batch system. Its default scheduler implements First-In First-Out (FIFO) and is not intended to directly implement complex site policy. SLURM does however provide a sufficiently sophisticated API for an external scheduler or meta-batch system to order its queues based upon site policy.

SLURM clusters are space shared with different jobs executing concurrently on (typically) different nodes. Multiple jobs may be allocated the same node(s) if the administrator has configured nodes for shared access and/or the job has requested shared resources for improved responsiveness. SLURM does not directly perform gang scheduling (time-slicing of parallel jobs). However it does does provide the infrastructure for a meta-scheduler to perform gang scheduling, namely an API to explicit preempt and later resume a job. An external scheduler may submit, signal, hold, reorder and terminate jobs via the API.

SLURM is not a meta-batch system like Globus[4] or DPCS (Distributed Production Control System)[8]. SLURM supports resource management across a single cluster.

SLURM is not a comprehensive cluster administration or monitoring package. While SLURM knows the state of its compute nodes, it makes no attempt to put this information to use in other ways, such as with a general purpose event logging mechanism or a back-end database for recording historical state. It is expected that SLURM will be deployed in a cluster with other tools performing these functions. 


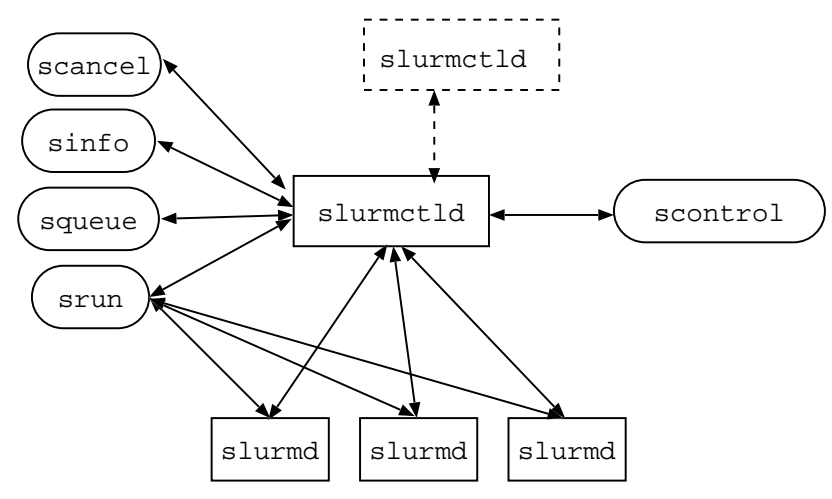

Figure 1: SLURM Architecture

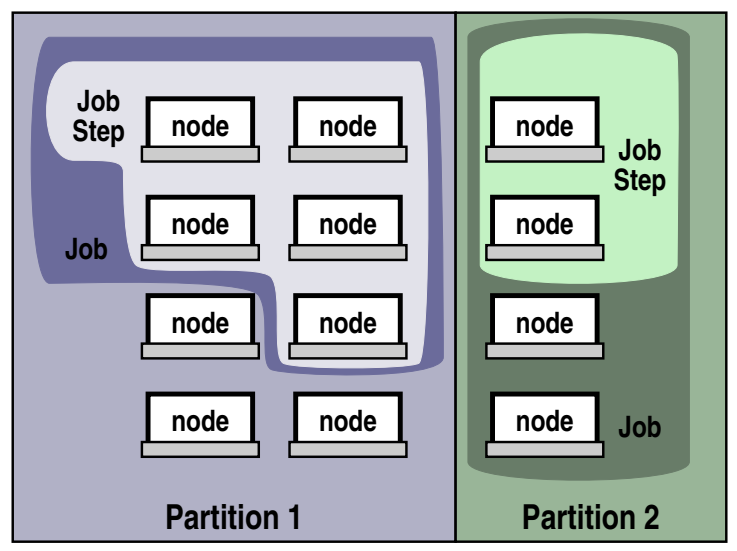

Figure 2: SLURM Entities

\subsection{Architecture}

As depicted in Figure 1, SLURM consists of a slurmd daemon running on each compute node, a central slurmctld daemon running on a management node (with optional fail-over twin), and five command line utilities: srun, scancel, sinfo, squeue, and scontrol, which can run anywhere in the cluster.

The entities managed by these SLURM daemons include nodes, the compute resource in SLURM, partitions, which group nodes into logical disjoint sets, jobs, or allocations of resources assigned to a user for a specified amount of time, and job steps, which are sets of parallel tasks within a job. Jobs are allocated nodes within partitions until the resources (nodes) within that partition are exhausted. Once a job is assigned a set of nodes, the user is able to initiate parallel work in the form of job steps in any configuration within the allocation. For instance a single job step may be started which utilizes all nodes allocated to the job, or several job steps may independently use a portion of the allocation. 


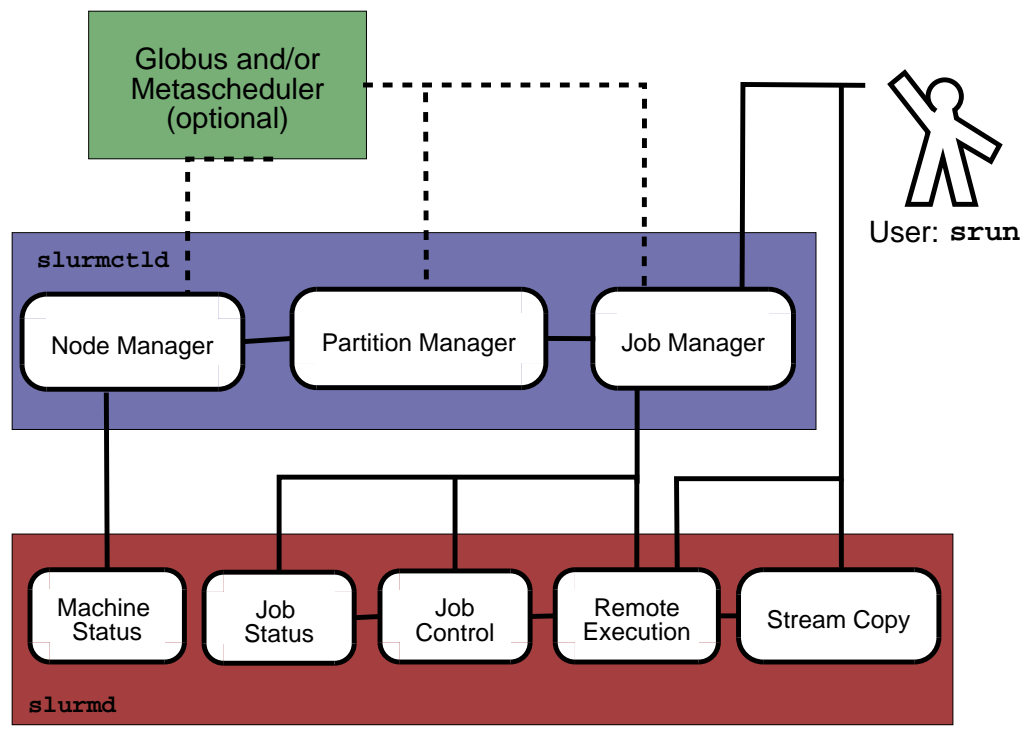

Figure 3: SLURM Architecture - Subsystems

Figure 2 further illustrates the interrelation of these entities as they are managed by SLURM. The diagram shows a group of compute nodes split into two partitions. Partition 1 is running one job, with one job step utilizing the full allocation of that job. The job in Partition 2 has only one job step using half of the original job allocation. That job might initiate additional job step(s) to utilize the remaining nodes of its allocation.

Figure 3 exposes the subsystems that are implemented within the slurmd and slurmctld daemons. These subsystems are explained in more detail below.

\subsubsection{Slurmd}

The slurmd running on each compute node can be compared to a remote shell daemon: it waits for work, executes the work, returns status, then waits for more work. It also asynchronously exchanges node and job status with slurmctld. The only job information it has at any given time pertains to its currently executing jobs. slurmd reads the common SLURM configuration file, /etc/slurm.conf, and has five major components:

- Machine and Job Status Services: Respond to controller requests for machine and job state information, and send asynchronous reports of some state changes (e.g. slurmd startup) to the controller. Job status includes $\mathrm{CPU}$ and real-memory consumption information for all processes including user processes, system daemons, and the kernel.

- Remote Execution: Start, monitor, and clean up after a set of processes (typically belonging to a parallel job) as dictated by the slurmctld dae- 
mon or an srun or scancel commands. Starting a process may include executing a prolog program, setting process limits, setting real and effective user id, establishing environment variables, setting working directory, allocating interconnect resources, setting core file paths, initializing the Stream Copy Service, and managing process groups. Terminating a process may include terminating all members of a process group and executing an epilog program.

- Stream Copy Service: Allow handling of stderr, stdout, and stdin of remote tasks. Job input may be redirected from a file or files, a srun process, or $/ \mathrm{dev} /$ null. Job output may be saved into local files or sent back to the srun command. Regardless of the location of stdout/err, all job output is locally buffered to avoid blocking local tasks.

- Job Control: Allow asynchronous interaction with the Remote Execution environment by propagating signals or explicit job termination requests to any set of locally managed processes.

\subsubsection{Slurmctld}

Most SLURM state information exists in the controller, slurmctld. When slurmctld starts, it reads the SLURM configuration file: /etc/slurm.conf. It also can read additional state information from a checkpoint file generated by a previous execution of slurmctld. slurmctld runs in either master or standby mode, depending on the state of its fail-over twin, if any. slurmctld has three major components:

- Node Manager: Monitors the state of each node in the cluster. It polls slurmd's for status periodically and receives state change notifications from slurmd daemons asynchronously. It ensures that nodes have the prescribed configuration before being considered available for use.

- Partition Manager: Groups nodes into non-overlapping sets called partitions. Each partition can have associated with it various job limits and access controls. The partition manager also allocates nodes to jobs based upon node and partition states and configurations. Requests to initiate jobs come from the Job Manager. scontrol may be used to administratively alter node and partition configurations.

- Job Manager: Accepts user job requests and can place pending jobs in a priority ordered queue. By default, the job priority is a simple sequence number providing FIFO ordering. An interface is provided for an external scheduler to establish a job's initial priority and API's are available to alter this priority through time for customers wishing a more sophisticated scheduling algorithm. The Job Manager is awakened on a periodical basis and whenever there is a change in state that might permit a job to begin running, such as job completion, job submission, partition up transition, node up transition, etc. The Job Manager then makes a pass through the 
priority ordered job queue. The highest priority jobs for each partition are allocated resources as possible. As soon as an allocation failure occurs for any partition, no lower-priority jobs for that partition are considered for initiation. After completing the scheduling cycle, the Job Manager's scheduling thread sleeps. Once a job has been allocated resources, the Job Manager transfers necessary state information to those nodes, permitting it to commence execution. Once executing, the Job Manager monitors and records the job's resource consumption (CPU time used, CPU time allocated, and real memory used) in near real-time. When the Job Manager detects that all nodes associated with a job have completed their work, it initiates clean-up and performs another scheduling cycle as described above.

\subsubsection{Command Line Utilities}

The command line utilities are the user interface to SLURM functionality. They offer users access to remote execution and job control. They also permit administrators to dynamically change the system configuration. The utilities read the global configuration, file /etc/slurm.conf, to determine the host(s) for slurmctld requests, and the ports for both for slurmctld and slurmd requests.

- scancel: Cancel a running or a pending job, subject to authentication. This command can also be used to sent an arbitrary signal to all processes associated with a job on all nodes.

- scontrol: Perform privileged administrative commands such as draining a node or partition in preparation for maintenance. Most scontrol functions can only be executed by privileged users.

- sinfo: Display a summary of partition and node information.

- squeue: Display the queue of running and waiting jobs and/or job steps. A wide assortment of filtering, sorting, and output format options are available.

- srun: Allocate resources, submit jobs to the SLURM queue, and initiate parallel tasks (job steps). Every set of executing parallel tasks has an associated srun process managing it. Jobs may be submitted for later execution (e.g. batch), in which case srun terminates after job submission. Jobs may also be submitted for interactive execution, where srun keeps running to shepherd the running job. In this case, srun negotiates connections with remote slurmd's for job initiation and to get stdout and stderr, forward $\operatorname{stdin}^{2}$, and respond to signals from the user. srun may also be instructed to allocate a set of resources and spawn a shell with access to those resources.

\footnotetext{
${ }^{2}$ srun command line options select the stdin handling method such as broadcast to all tasks, or send only to task 0 .
} 


\subsubsection{Communications Layer}

SLURM uses Berkeley sockets for communications. At LLNL we are using an Ethernet for SLURM communications and the Quadrics Elan switch exclusively for user applications. The SLURM configuration file permits the identification of each node's name to be used for communications as well as its hostname. In the case of a control machine known as mcri to be communicated with using the name emcri this is represented in the configuration file as ControlMachine $=m c r i$ ControlAddr=emcri.

While SLURM is able to over 1000 nodes without difficulty using sockets on an Ethernet, we are reviewing other communication mechanisms which may offer improved scalability. One possible alternative is STORM[1]. STORM uses the cluster interconnect and Network Interface Cards to provide high-speed communications including a broadcast capability. STORM only supports the Quadrics Elan interconnnect at present, but does offer the promise of improved performance and scalability.

Internal SLURM functions pack and unpack data structures in machine independent format. We considered the use of XML style messages, but felt this would adversely impact performance (albeit slightly). If XML support is desired, it is straightforward to perform a translation.

\subsubsection{Security}

SLURM has a simple security model: Any user of the cluster may submit parallel jobs to execute and cancel his own jobs. Any user may view SLURM configuration and state information. Only privileged users may modify the SLURM configuration, cancel any job, or perform other restricted activities. Privileged users in SLURM include the users root and SlurmUser (as defined in the SLURM configuration file). If permission to modify SLURM configuration is required by others, set-uid programs may be used to grant specific permissions to specific users.

We presently support two authentication mechanisms: authd[2] and munged. Both are quite similar and the munged implementation is described below. Trust between SLURM components and utilities is established through use of communication-layer encryption. A munged daemon running as user root on each node confirms the identify of the user making the request and generates a credential. The credential contains a user id, group id, time-stamp, lifetime, some pseudo-random information, and any user supplied information. munged uses a private key to generate a Message Authentication Code (MAC) for the credential. munged then uses a public key to symmetrically encrypt the credential including the MAC. SLURM daemons and programs transmit this encrypted credential with communications. The SLURM daemon receiving the message sends the credential to munged on that computer. munged decrypts the credential using its private key, validates it and returns the user id and group id of the user originating the credential. munged prevents replay of a credential on any single node by recording authenticated credentials. The user supplied 
information can include node identification information to prevent a credential from being used on nodes it is not destined for.

When resources are allocated to a user by the controller, a "job credential" is generated by combining the user id, the list of resources allocated (nodes and processors per node), and the credential lifetime. This "job credential" is encrypted with a slurmctld private key. This credential is returned to the requesting agent along with the allocation response, and must be forwarded to the remote slurmd's upon job initiation. slurmd decrypts this credential with the slurmctld's public key to verify that the user may access resources on the local node. slurmd also uses this "job credential" to authenticate standard input, output, and error communication streams. The "job credential" differs from the munged credential in that it always contains a list of nodes and is explicitly revoked by slurmctld upon job termination.

Both slurmd and slurmctld also support the use of Pluggable Authentication Modules (PAM) for additional authentication beyond communication encryption and job credentials. Specifically if a job credential is not forwarded to slurmd on a job initiation request, slurmd may execute a PAM module. The PAM module may authorize the request based upon methods such as a flat list of users or an explicit request to the SLURM controller. slurmctld may use PAM modules to authenticate users based upon UNIX passwords, Kerberos, or any other method that may be represented in a PAM module.

Access to partitions may be restricted via a "RootOnly" flag. If this flag is set, job submit or allocation requests to this partition are only accepted if the effective user ID originating the request is a privileged user. The request from such a user may submit a job as any other user. This may be used, for example, to provide specific external schedulers with exclusive access to partitions. Individual users will not be permitted to directly submit jobs to such a partition, which would prevent the external scheduler from effectively managing it.

Access to partitions may also be restricted to users who are members of specific Unix groups using a "AllowGroups" specification.

\subsection{Example: Executing a Batch Job}

In this exampe a user wishes to run a job in batch mode, in which srun returns immediately and the job executes "in the background" when resources are available. The job is a two-node run of script containing mping, a simple MPI application. The user submits the job:

srun --batch --nodes 2 --nprocs 2

The script myscript contains:

srun hostname

mping 11048576 
The srun command authenticates the user to the controller and submits the job request. The request includes the srun environment, current working directory, and command line option information. By default, stdout and stderr are sent to files in the current working directory and stdin is copied from /dev/null.

The controller consults the partition manager to test whether the job will ever be able to run. If the user has requested a non-existent partition, more nodes than are configured in the partition, a non-existent constraint, etc., the partition manager returns an error and the request is discarded. The failure is reported to srun which informs the user and exits, for example:

srun: error: Unable to allocate resources: Invalid partition name

On successful submission, the controller assigns the job a unique slurm id, adds it to the job queue and returns the slurm id to srun which reports this to user and exits, returning success to the user's shell:

srun: jobid 42 submitted

The controller awakens the Job Manager which tries to run jobs starting at the head of the priority ordered job queue. It finds job 42 and makes a successful request to the partition manager to allocate two nodes from the default (or requested) partition: $\operatorname{dev} 6$ and $\operatorname{dev} \%$.

The Job Manager then sends a request to the slurmd on the first node in the job dev6 to initiate a srun of the user's command line ${ }^{3}$. The Job Manager also sends a copy of the environment, current working directory, stdout and stderr location, along with other options. Additional environment variables are appended to the user's environment before it is sent to the remote slurmd detailing the job's resources, such as the slurm job id (42) and the allocated nodes $(\operatorname{dev}[6-7])$.

The remote slurmd establishes the new environment, executes a SLURM prolog program (if one is configured) as user root, and executes the job script (or command) as the submitting user. The srun within the job script detects that it is running with allocated resources from the presence of the SLURM_JOBID environment variable. srun connects to slurmctld to request a "job step" to run on all nodes of the current job. slurmctld validates the request and replies with a job credential and switch resources. srun then contacts slurmd's running on both dev6 and dev7, passing the job credential, environment, current working directory, command path and arguments, and interconnect information. The slurmd's verify the valid job credential, connect stdout and stderr back to srun, establish the environment, and execute the command as the submitting user.

Unless instructed otherwise by the user, stdout and stderr are copied to files in the current working directory by srun:

/path/to/cwd/slurm-42. out

/path/to/cwd/slurm-42. err

\footnotetext{
${ }^{3}$ Had the user submitted a job script, this script would be initiated on the first node allocated to the job
} 
The user may examine the output files at any time if they reside in a globally accessible directory. In this example slurm-42. out would contain the output of the job script's two commands (hostname and mping):

$\begin{array}{lllll}\text { dev6 } & & & & \\ \text { dev7 } & & & & \\ 1 \text { pinged } & 0: & 1 \text { bytes } & 5.38 \mathrm{uSec} & 0.19 \mathrm{MB} / \mathrm{s} \\ 1 \text { pinged } & 0: & 2 \text { bytes } & 5.32 \mathrm{uSec} & 0.38 \mathrm{MB} / \mathrm{s} \\ 1 \text { pinged } & 0: & 4 \text { bytes } & 5.27 \mathrm{uSec} & 0.76 \mathrm{MB} / \mathrm{s} \\ 1 \text { pinged } & 0: & 8 \text { bytes } & 5.39 \mathrm{uSec} & 1.48 \mathrm{MB} / \mathrm{s} \\ \ldots & & & & \\ 1 \text { pinged } & 0: & 1048576 \text { bytes } & 4682.97 \mathrm{uSec} & 223.91 \mathrm{MB} / \mathrm{s}\end{array}$

When the tasks complete execution srun is notified by slurmd of each task's exit status. srun reports job step completion to the Job Manager and exits. slurmd detects when the job script terminates and notifies the Job Manager of its exit status and begins cleanup. The Job Manager directs the slurmd's formerly assigned to the job to run the SLURM epilog program. Finally, the Job Manager releases the resources allocated to job 42 and updates the job status to complete. The record of a job's existence is eventually purged.

\subsection{Example: Executing an Interactive Job}

In this example a user wishes to run the same mping command in interactive mode, in which srun blocks while the job executes and stdout/stderr of the job are copied onto stdout/stderr of srun. The user submits the job, this time requesting interactive execution:

srun --nodes 2 --nprocs 2 mping 11048576

The srun command authenticates the user to the controller and makes a request for a resource allocation and job step. The Job Manager responds with a list of nodes, a job credential, and interconnect resources on successful allocation. If resources are not immediately available, the request terminates or block depending upon user option.

If the request is successful, srun forwards the job run request to the assigned slurmd 's in the same manner as the srun in the batch job script. In this case, the user sees the program output on stdout of srun:

$\begin{array}{llrrr}1 \text { pinged } & 0: & 1 \text { bytes } & 5.38 \mathrm{uSec} & 0.19 \mathrm{MB} / \mathrm{s} \\ 1 \text { pinged } & 0: & 2 \text { bytes } & 5.32 \mathrm{uSec} & 0.38 \mathrm{MB} / \mathrm{s} \\ 1 \text { pinged } & 0: & 4 \text { bytes } & 5.27 \mathrm{uSec} & 0.76 \mathrm{MB} / \mathrm{s} \\ 1 \text { pinged } & 0: & 8 \text { bytes } & 5.39 \mathrm{uSec} & 1.48 \mathrm{MB} / \mathrm{s} \\ \ldots & & & & \\ 1 \text { pinged } & 0: & 1048576 \text { bytes } & 4682.97 \mathrm{uSec} & 223.91 \mathrm{MB} / \mathrm{s}\end{array}$

When the job terminates, srun receives an EOF on each stream and closes it, then receives the task exit status from each slurmd. The srun process notifies 
slurmctld that the job is complete and terminates. The controller contacts all slurmd's allocated to the terminating job and issues a request to run the SLURM epilog, then releases the job's resources.

If a signal is received by srun while the job is executing (for example, a SIGINT resulting from a Control-C), it is sent to each slurmd which terminates the individual tasks and reports this to the job status manager, which cleans up the job.

\section{Controller Design}

The controller is modular and multi-threaded. Independent read and write locks are provided for the various data structures for scalability. Full controller state information is written to disk periodically with incremental changes written to disk immediately for fault tolerance. Since the controller does not need to execute as user root, we recommend a special account be established for this purpose. The user name of this account should be recorded as SlurmUser in the configuration file so that slurmd authorizes its requests. The controller includes the following subsystems: Node Manager, Partition Manager, and Job Manager. Each of these subsystems is described in detail below.

\subsection{Node Management}

Job ManagerJob ManagerJob ManagerThe node manager monitors the state of nodes. Node information monitored includes:

- Count of processors on the node

- Size of real memory on the node

- Size of temporary disk storage

- State of node (RUN, IDLE, DRAINED, etc.)

- Weight (preference in being allocated work)

- Feature (arbitrary description)

- IP address

The SLURM administrator can specify a list of system node names using a regular expression in the SLURM configuration file or in the SLURM tools (e.g. "NodeName $=\operatorname{linux}[001-512] \mathrm{CPUs}=4$ RealMemory $=1024$ TmpDisk $=4096$ Weight=4 Feature=Linux"). These values for CPUs, RealMemory, and TmpDisk are considered the minimal node configuration values which are acceptable for the node to enter into service. The slurmd registers whatever resources actually exist on the node and this is recorded by the Node Manager. Actual node resources are checked on slurmd initialization and periodically thereafter. If a node registers with less resources than configured, it is placed in $D O W N$ 
state and the event logged. Otherwise the actual resources reported are recorded and possibly used as a basis for scheduling (e.g. if the node has more RealMemory than recorded in the configuration file, the actual node configuration may be used for determining suitability for any application, alternately the data in the configuration file may be used for possibly improved scheduling performance). Note the regular expression node name syntax permits even very large heterogeneous clusters to be described in only a few lines. In fact, a smaller number of unique configurations can provide SLURM with greater efficiency in scheduling work.

The weight is used to order available nodes in assigning work to them. In a heterogeneous cluster, more capable nodes (e.g. larger memory or faster processors) should be assigned a larger weight. The units are arbitrary and should reflect the relative value of each resource. Pending jobs are assigned the least capable nodes (i.e. lowest weight) which satisfy their requirements. This tends to leave the more capable nodes available for those jobs requiring those capabilities.

The feature is an arbitrary string describing the node, such as a particular software package, file system, or processor speed. While the feature does not have a numeric value, one might include a numeric value within the feature name (e.g. "1200MHz" or "16GB_Swap"). If the nodes on the cluster have disjoint features (e.g. different "shared" file systems), one should identify these as features (e.g. "FS1", "FS2", etc.). Programs may then specify that all nodes allocated to it should have the same feature, but that any of the specified features is acceptable (e.g. "Feature $=F S 1|F S 2| F S 3$ " means the job should be allocated nodes that all have the feature "FS1" or they all have feature "FS2", etc.).

Node records are kept in an array with hash table lookup. If nodes are given names containing sequence numbers (e.g. "lx01", "lx02", etc.), the hash table permits specific node records to be located very quickly and this is our recommended naming convention for larger clusters.

An API is available to view any of this information and to update some node information (e.g. state). APIs designed to return SLURM state information permit the specification of a time-stamp. If the requested data has not changed since the time-stamp specified by the application, the application's current information need not be updated. The API returns a brief "no_change" response rather than returning relatively verbose state information. Changes in node configurations (e.g. node count, memory, etc.) or the nodes actually in the cluster should be reflected in the SLURM configuration files. SLURM configuration may be updated without disrupting jobs that are currently executing.

\subsection{Partition Management}

The partition manager identifies groups of nodes to be used for execution of user jobs. One might consider this the actual resource scheduling component. Data associated with a partition includes: 
- Name

- RootOnly flag to indicated that only users root or SlurmUser may initiate jobs (for any user)

- List of associated nodes (may use regular expression)

- State of partition (UP or DOWN)

- Maximum time limit for any job

- Maximum nodes allocated to any single job

- List of groups permitted to use the partition (defaults to ALL)

- Shared access (YES, NO, or FORCE)

- Default partition (if not specified in job request)

It is possible to alter most of this data in real-time in order to effect the scheduling of pending jobs (currently executing jobs would not be effected). This information is confined to the slurmctld machine for better scalability. It is used by the Job Manager (and possibly an external scheduler), which either exist only on the control machine or communicate only with the control machine.

The nodes in a partition may be designated for exclusive or non-exclusive use by a job. A shared value of "YES" indicates that jobs may share nodes upon request. A shared value of "NO" indicates that jobs are always given exclusive use of allocated nodes. A shared value of "FORCE" indicates that jobs are never ensured exclusive access to nodes, but SLURM may initiate multiple jobs on the nodes for high system utilization and responsiveness. In this case, job requests for exclusive node access are honored. Non-exclusive access may negatively impact the performance of parallel jobs or cause them to fail upon exhausting shared resources (e.g. memory or disk space). However, shared resources may improve overall system utilization and responsiveness. The proper support of shared resources, including enforcement of limits on these resources, entails a substantial amount effort which we are not presently planning to address. However we have designed SLURM so as to not preclude the addition of such a capability at a later time if so desired. Future enhancements could include constraining jobs to a specific CPU count or memory size within a node, which could be used to effectively space-share individual node. The partition manager will allocate nodes to pending jobs upon request by the Job Manager.

Submitted jobs can specify desired partition, CPU count, node count, task count, the need for contiguous nodes assignment, and (optionally) an explicit list of nodes. Nodes are selected so as to satisfy all job requirements. For example a job requesting four CPUs and four nodes will actually be allocated eight CPUs and four nodes in the case of all nodes having two CPUs each. The request may also indicate node configuration constraints such as minimum real memory or CPUs per node, required features, etc. 
Nodes are selected for possible assignment to a job based upon it's configuration requirements (e.g. partition specification, minimum memory, temporary disk space, features, node list, etc.). The selection is refined by determining which nodes are up and available for use. Groups of nodes are then considered in order of weight, with the nodes having the lowest weight preferred. Finally the physical location of the nodes is considered.

Bit maps are used to indicate which nodes are up, idle, associated with each partition, and associated with each unique configuration. This technique permits scheduling decisions to normally be made by performing a small number of tests followed by fast bit map manipulations. If so configured, a job's resource requirements would be compared against the (relatively small number of) node configuration records, each of which has an associated bit map. Usable node configuration bitmaps would be ANDed with the selected partitions bit map ANDed with the UP node bit map and possibly ANDed with the IDLE node bit map (this last test depends upon the desire to share resources). This method can eliminated tens of thousands of node configuration comparisons that would otherwise be required in large heterogeneous clusters.

The actual selection of nodes for allocation to a job is currently tuned for the Quadrics interconnect. This hardware supports hardware message broadcast, but only if the nodes are contiguous. If a job is not allocated contiguous nodes, a slower software based multi-cast mechanism is used. Jobs will be allocated continuous nodes to the extent possible (in fact, contiguous node allocation can be required by a job if so specified at submission time). If contiguous nodes can not be allocated to a job, it will be allocated resources from the minimum number of sets of contiguous nodes possible. If multiple sets of contiguous nodes can be allocated to a job, the one which most closely fits the job's requirements will be used. This technique will leave the largest continuous sets of nodes intact for jobs requiring them.

The partition manager buildw a list of nodes to satisfy a job's request. It also cachew the IP addresses of each node and provide this information to srun at job initiation time for improved performance.

The failure of any node to respond to the partition manager only effects jobs associated with that node. In fact, jobs may indicate they should continue executing even if nodes allocated to it cease responding. In this case, the job needs to provide for its own fault-tolerance. All other jobs and nodes in the

cluster will continue to operate after a node failure. No additional work is allocated to the failed node and it will be pinged periodically to determine when it is responding. The node may then be returned to service (depending upon the ReturnToService value in the SLURM configuration.

\subsection{Configuration}

A single configuration file applies to all SLURM daemons and commands. Most of this information is used only be the controller. Only the host and port information is used by most commands. A sample configuration file follows. 


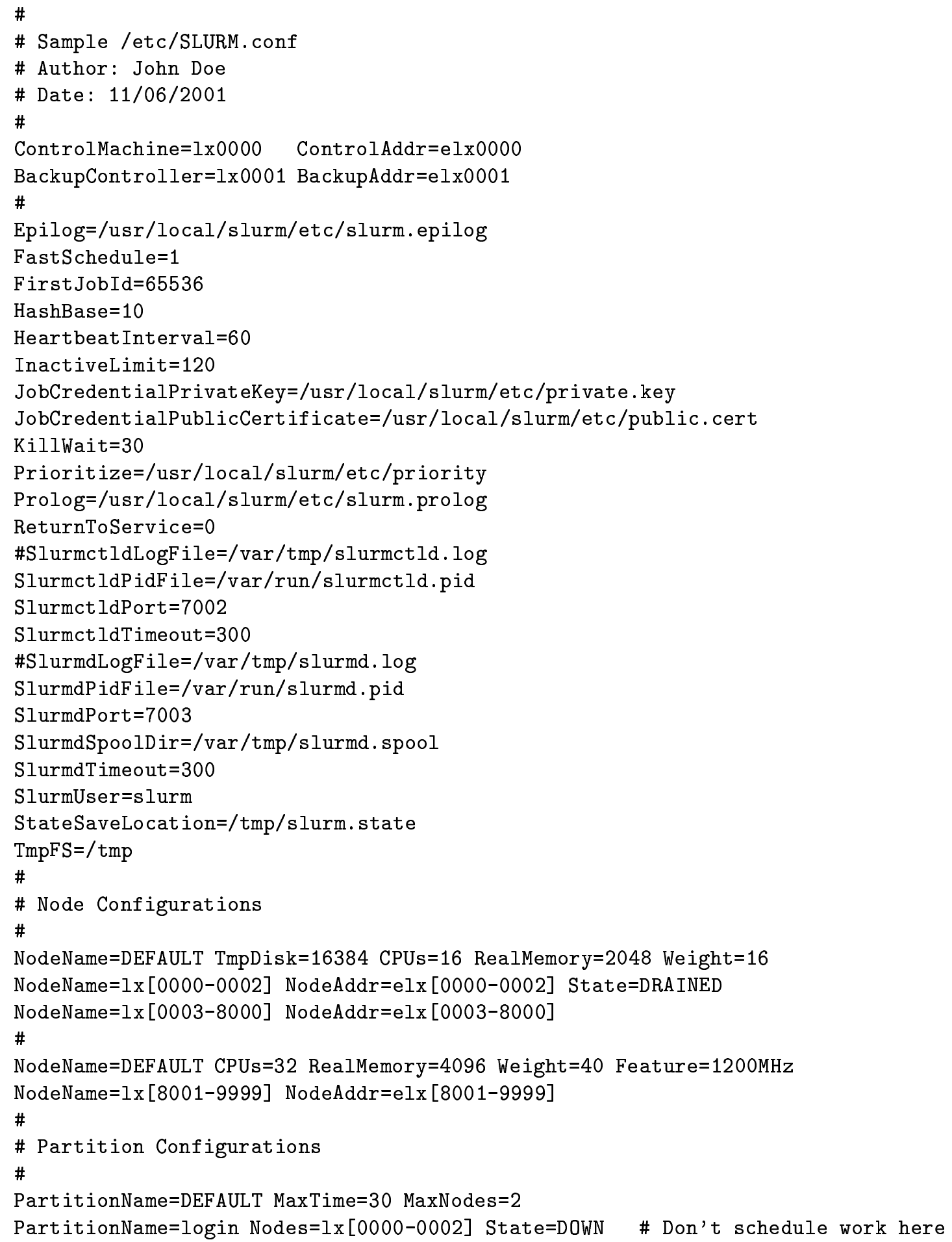




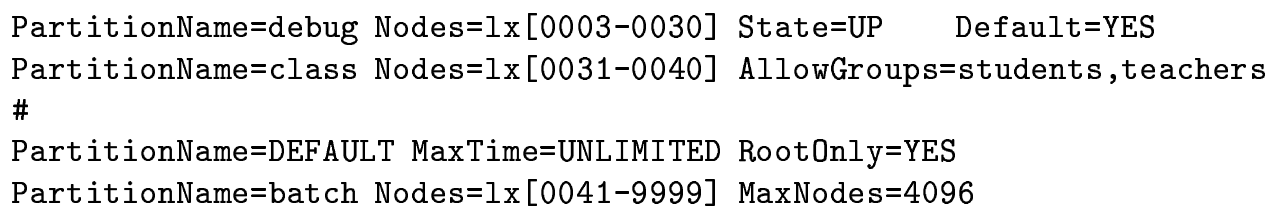

\subsection{Job Manager}

There are a multitude of parameters associated with each job:

- Job name

- User ID

- Job ID

- Working Directory

- Partition

- Priority

- Node constraints (processors, memory, features, etc.)

- and many more

Job records have an associated hash table for rapidly locating specific records. They also have bit maps of requested and/or allocated nodes (as described above).

The core functions supported by the Job Manager include:

- Request resource request (job may be queued)

- Reset priority of jobs (for external scheduler to order queue)

- Status job (including node list, memory and CPU use data)

- Signal job (send arbitrary signal to all processes associated with a job)

- Terminate job (remove all processes)

- Preempt/resume job (future)

- Checkpoint/restart job (future)

- Change node count of running job (could fail if insufficient resources are available, future) 
Jobs are placed in a priority ordered queue and allocated nodes as selected by the Partition Manager. SLURM implements a very simple scheduling algorithm, namely FIFO. An attempt is made to schedule pending jobs on a periodic basis and whenever any change in job, partition, or node state might permit the scheduling of a job. All nodes allocated to a job remain so until all processes associated with that job terminate. If a node allocated to a job fails, the job may either continue execution or terminate depending upon its configuration.

We are well aware this scheduling algorithm does satisfy the needs of many customers and provide the means for establishing other scheduling algorithms. Before a newly arrived job is placed into the queue, it is assigned a priority that may be established by an administrator defined program (Prioritize in the configuration file). SLURM APIs permit an external entity to alter the priorities of jobs at any time to re-order the queue as desired. The Maui Scheduler[6, 9] is one example of an external scheduler suitable for use with SLURM.

Another scheduler that we plan to offer with SLURM is DPCS[8]. DPCS has flexible scheduling algorithms that suit our needs well and provides the scalability required for this application. Most of the resource accounting and some of the job management functions presently within DPCS would be moved into the proposed SLURM Job Management component. DPCS does require some modification to operate within this new, richer environment.

The Job Manager collects resource consumption information (CPU time used, CPU time allocated, and real memory used) associated with a job from the slurmd daemons. When a job approaches its time limit (as defined by wall-clock execution time) or an imminent system shutdown has been scheduled, the job is terminated. The actual termination process is to notify slurmd daemons on nodes allocated to the job of the termination request along with a time period in which to complete the termination. The slurmd job termination procedure, including job signaling, is described in the slurmd section.

If for some reason, there are non-killable processes associated with the job, nodes associated with those processes are drained and the other nodes relinquished for other uses.

One may think of a "job" as described above as an allocation of resource and a user script rather than a collection of parallel tasks. For that, the scripts execute srun commands to initiate the parallel tasks or "job steps". The job may include multiple job steps, executing sequentially and or concurrently either on separate or overlapping nodes. Job steps have associated with them specific nodes (some or all of those associated with the job), tasks, and a task distribution (cyclic or block) over the nodes.

The management of job steps is considered a component of the job manager. Supported job step functions include:

- Register Job Step

- Get Job Step Information

- Run Job Step Request 
- Signal Job Step

Job step information includes a list of nodes (entire set or subset of those allocated to the job) and a credential used to bind communications between the tasks across the interconnect. The slurmctld constructs this credential, distributes it the the relevant slurmd daemons, and sends it to the srun initiating the job step.

\subsection{Fault Tolerance}

A backup controller, if one is configured, periodically pings the primary controller. Should the primary controller cease responding, the backup loads state information from the last controller state save, and assumes control. All slurmd daemons are notified of the new controller location and requested to upload current state information to it. When the primary controller is returned to service, it tells the backup controller to save state and terminate. The primary then loads state, assumes control, and notifies slurmd daemons.

SLURM utilities and the APIs read the /etc/slurmd.conf and initially try to contact the primary slurmctld. Should that attempt fail, an attempt is made to contact the backup slurmctld before terminating.

\section{Slurmd}

The slurmd daemon is a multi-threaded daemon for managing user jobs and monitoring system state. Upon initiation it reads the configuration file, captures system state, attempts an initial connection to the SLURM controller, and awaits requests. It services requests for system state, accounting information, job initiation, job state, job termination, and job attachment. On the local node it offers an API to translate local process ID's into SLURM job id's.

It's most common action is to report system state upon request. Upon slurmd startup and periodically thereafter, it gathers the processor count, real memory size, and temporary disk space for the node. Should those values change, the controller is notified. Another thread is created to capture CPU, real-memory and virtual-memory consumption from the process table entries. Differences in resource utilization values from one process table snapshot to the next are accumulated. slurmd insures these accumulated values are not decremented if resource consumption for a user happens to decrease from snapshot to snapshot, which would simply reflect the termination of one or more processes. Both the real and virtual memory high-water marks are recorded and the integral of memory consumption (e.g. megabyte-hours). Resource consumption is grouped by user ID and SLURM job ID (if any). Data is collected for system users (root, ftp, ntp, etc.) as well as customer accounts. The intent is to capture all resource use including kernel, idle and down time. Upon request, the accumulated values are uploaded to slurmctld and cleared.

slurmd accepts requests from srun and slurmctld to initiate and terminate user jobs. The initiate job request contains: real and effective user IDs, envi- 
ronment variables, working directory, task numbers, job credential, interconnect specifications and authorization, core paths, SLURM job id, and the command line to execute. System specific programs can be executed on each allocated node prior to the initiation of a user job and after the termination of a user job (e.g. Prolog and Epilog in the configuration file). These programs are executed as user root and can be used to establish an appropriate environment for the user (e.g. permit logins, disable logins, terminate "orphan" processes, etc.). slurmd executes the Prolog program, resets its session ID, and then initiates the job as requested. It records to disk the SLURM job ID, session ID, process ID associated with each task, and user associated with the job. In the event of slurmd failure, this information is recovered from disk in order to identify a specific job. This job identity is used in communications with the SLURM controller.

The job termination request contains the SLURM job ID and a delay period. Jobs have an API made available to register with slurmd exactly which process(s) should be sent what signals how long before termination. slurmd sends the requested signal (or SIGNXCPU if none specified) to the identified process(es) associated with the SLURM job (or all processes associated with that session ID or process tree by default), sleep for the delay specified, and send SIGKILL to all of the job's processes. If the processes do not terminate, SIGKILL is sent again. If the processes still do not terminate slurmd notifies the slurmctld, which logs the event and sets node's state to DRAINED. After all processes terminate, slurmd executes the epilog program (if any).

\section{Command Line Utilities}

\section{1 scancel}

scancel terminates queued or running jobs or job steps. If the job is queued, it is just removed. If the job is running, it is signaled and terminated as described in the slurmd section of this document. It identifies the job(s) to be terminated through user specification of: SLURM job ID, job step ID, user name, partition name, and/or job state. If a job ID is supplied, all job steps associated with the job are terminated as well as the job and its resource allocation. If a job ID and job step ID are supplied, only that job step is terminated. scancel can only be executed by the job's owner or a privileged user.

\section{2 scontrol}

scontrol is a tool meant for SLURM administration by user root. It provides the following capabilities:

- Shutdown - Cause slurmctld to save state and terminate.

- Reconfigure - Cause slurmctld to re-read its configuration file. 
- Show configuration parameters - Display the values of general SLURM configuration parameters such as locations of files and values of timers.

- Show job state - Display the state information of a particular job or all jobs in the system.

- Show job step state - Display the state information of a particular job step or all job steps in the system.

- Show node state - Display the state and configuration information of a particular node, a set of nodes (using regular expressions to identify their names), or all nodes.

- Show partition state - Display the state and configuration information of a particular partition or all partitions.

- Update job state - Update the state information of a particular job in the system. Note that not all state information can be changed in this fashion (e.g. the nodes allocated to a job).

- Update node state - Update the state of a particular node. Note that not all state information can be changed in this fashion (e.g. the amount of memory configured on a node). In some cases, you may need to modify the SLURM configuration file and cause it to be re-read using the "Reconfigure" command described above.

- Update partition state - Update the state of a partition node. Note that not all state information can be changed in this fashion (e.g. the default partition). In some cases, you may need to modify the SLURM configuration file and cause it to be re-read using the "Reconfigure" command described above.

\section{3 squeue}

squeue reports the state of SLURM jobs. It can filter these jobs input specification of job state (RUN, PENDING, etc.), job ID, user name, job name, etc. If no specification is supplied, the state of all jobs is reported. squeue also has a variety of sorting and output options.

\section{4 sinfo}

sinfo reports the state of SLURM partitions and nodes. By default, it reports a summary of partition state with node counts and a summary of the configuration of those nodes (e.g. "PartitionName=batch Nodes $=\mathrm{lx}[1000-9999]$ RealMemory $=2048-4096$ IdleNodes $=1234 \ldots$...). A variety of output formatting options exist. 


\section{5 srun}

srun is the user interface to accessing resources managed by SLURM. Users may utilize srun to allocate resources, submit batch jobs, run jobs interactively, attach to currently running jobs or launch a set of parallel tests (job step) for a running job. srun supports a full range of options to specify job constraints and characteristics, for example minimum real memory, temporary disk space, and cpus per node, as well as time limits, stdin/stdout/stderr handling, signal handling, and working directory for job. The full range of options are detailed in table 1.

\begin{tabular}{lcl}
\hline Option & Arg type & Description \\
\hline attach & string & attach srun to a running job \\
allocate & boolean & allocate nodes only \\
batch & boolean & submit a batch script to job queue \\
cddir & string & working directory of remote processes \\
constraint & string & arbitrary feature constraints \\
contiguous & boolean & allocate contiguous nodes only \\
cpus-per-task & number & number of cpus needed per process. \\
distribution & string & distribution method for processes (block|cyclic) \\
error & string & location of stderr redirection \\
immediate & boolean & exit if resources are not immediately available \\
input & string & location of stdin redirection \\
job-name & string & name of job \\
kill-off & boolean & do not terminate job on node value \\
label & boolean & prepend task number to lines of stdout/err \\
mem & number & minimum amount of real memory per node \\
mincpus & number & minimum number of cpus per node \\
nodelist & string & request a specific set of hosts \\
nodes & number & number of nodes on which to run \\
ntasks & number & number of tasks to run \\
output & string & location of stdout redirection \\
overcommit & boolean & allow more than 1 process per cpu \\
partition & string & partition name in which to run \\
share & boolean & allow nodes to be shared with other jobs \\
time & number & wall clock time limit in minutes \\
tmp & number & minimum amount of temporary disk space \\
verbose & boolean & verbose operation \\
version & boolean & print srun version and exit \\
\hline & &
\end{tabular}

Table 1: srun options

The srun utility can run in four different modes: interactive, in which the srun process remains resident in the user's session, manages stdout/stderr/stdin and forwards signals to the remote tasks, batch in which srun submits a job script to the SLURM queue for later execution, allocate in which srun requests 


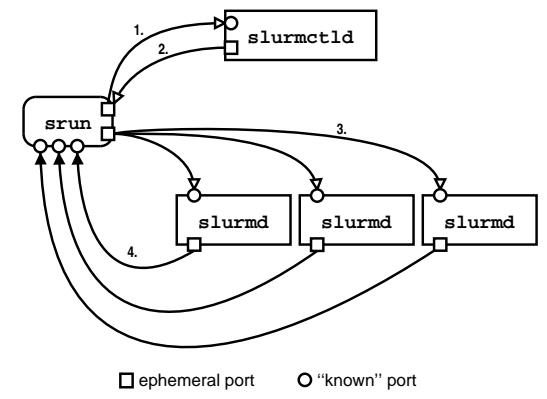

Figure 4: Job initiation connections overview. 1. srun connects to slurmctld requesting resources. 2. slurmctld issues a response, with list of nodes and job credential. 3 . srun opens a listen port for every task in the job step, then sends a run job step request to slurmd. 4. slurmd's initiate job step and connect back to srun for stdout/err.

resources from the SLURM controller and spawns a shell with access to those resources, and finally attach in which srun attaches to a currently running job and display stdout/stderr in real time from the remote tasks, forward signals from the user's terminal and so on.

An interactive job may also be forced into the "background" with a special control sequence typed at the user's terminal. This sequence causes another srun to attach to the running job while the interactive srun terminates. Output from the running job is subsequently redirected to files in the current working directory and stdin is copied from /dev/null. A backgrounded job may be reattached to a user's terminal at a later time by running

srun --attach jobid

at any time, though the remote srun is not terminated as the result of an attach.

\section{$5 \quad$ Job Initiation Design}

There are three modes in which jobs may be run by users under SLURM. The first and most simple is interactive mode, in which stdout and stderr are displayed on the user's terminal in real time, and stdin and signals may be forwarded from the terminal transparently to the remote tasks. The second is batch or queued mode, in which the job is queued until the request for resources can be satisfied, at which time the job is run by SLURM as the submitting user. In allocate mode, a job is allocated to the requesting user, under which the user may manually run job steps via a script or in a subshell spawned by srun.

Figure 4 gives a high-level depiction of the connections that occur between SLURM components during a general interactive job startup. srun requests resources from the slurmctld, which responds with the list of allocated nodes, timelimit, job credential, etc. if the request is granted. srun then initializes listen ports for each task and sends a message to the slurmd's on the allocated 


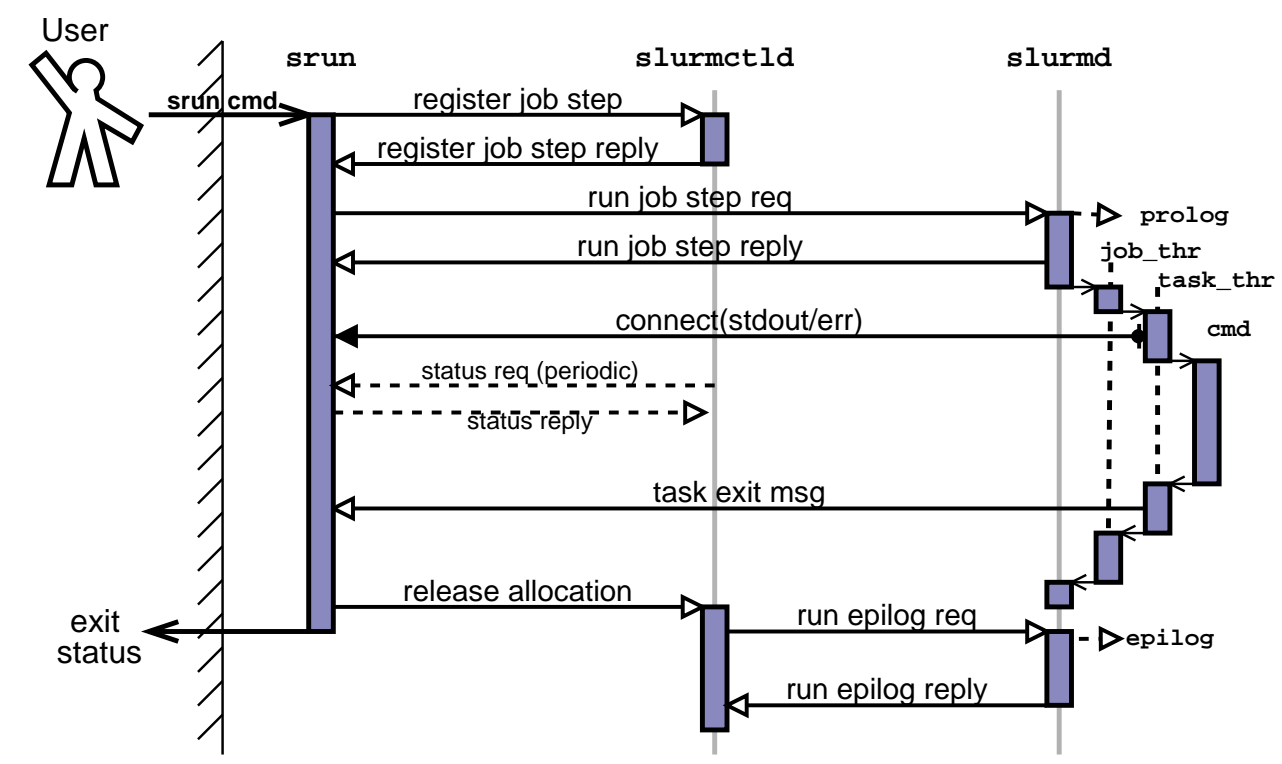

Figure 5: Interactive job initiation. srun simultaneously allocates nodes and a job step from slurmctld then sends a run request to all slurmd's in job. Dashed arrows indicate a periodic request that may or may not occur during the lifetime of the job.

nodes requesting that the remote processes be initiated. The slurmd's begin execution of the tasks and connect back to srun for stdout and stderr. This process and the other initiation modes are described in more detail below.

\subsection{Interactive job initiation}

Interactive job initiation is illustrated in figure 5 . The process begins with a user invoking srun in interactive mode - in figure 5 , the user has requested an interactive run of the executable "cmd" in the default partition.

After processing command line options, srun sends a message to slurmctld registering a job step. This message simultaneously requests an allocation (or job) and a job step. srun waits for a reply from slurmctld, which may not come instantly if the user has requested that srun block until resources are available. When resources are available for the user's job, slurmctld replies with a job credential, list of nodes that were allocated, cpus per node, and so on. srun then sends a message each slurmd on the allocated nodes requesting that a job step be initiated. The slurmd's verify that the job is valid using the forwarded job credential and then respond to srun.

Each slurmd invokes a job thread to handle the request, which in turn invokes a task thread for each requested task. The task thread connects back to a port opened by srun for stdout and stderr. The host and port for this connection is contained in the run request message sent to this machine by 


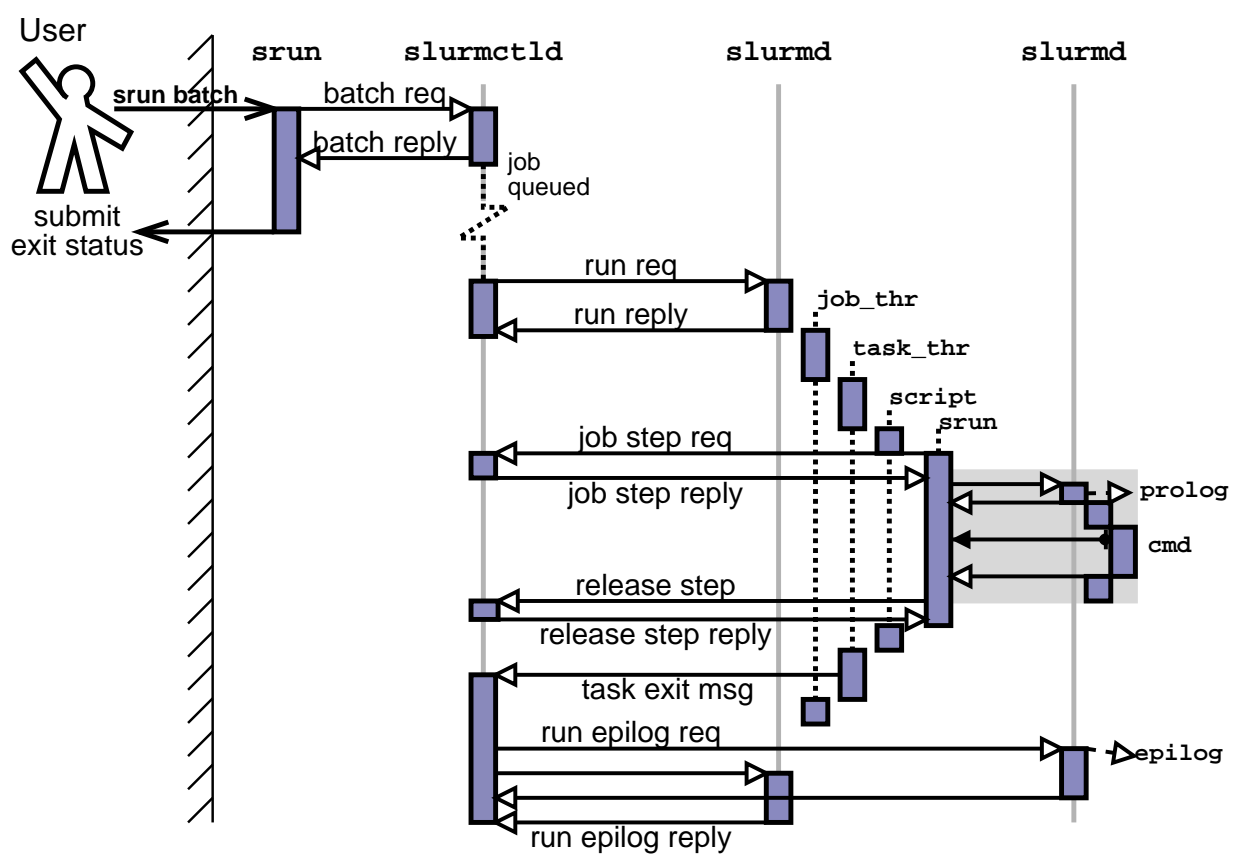

Figure 6: Queued job initiation. slurmctld initiates the user's job as a batch script on one node. Batch script contains an srun call which initiates parallel tasks after instantiating job step with controller. The shaded region is a compressed representation and is illustrated in more detail in the interactive diagram (figure 5).

srun. Once stdout and stderr have successfully been connected, the task thread takes the necessary steps to initiate the user's executable on the node, initializing environment, current working directory, and interconnect resources if needed.

Once the user process exits, the task thread records the exit status and sends a task exit message back to srun. When all local processes terminate, the job thread exits. The srun process either waits for all tasks to exit, or attempt to clean up the remaining processes when a single task exits based upon user option. Regardless, once all tasks are finished, srun sends a message to the slurmctld releasing the allocated nodes, then exits with an appropriate exit status.

When the slurmctld receives notification that srun no longer needs the allocated nodes, it issues a request for the epilog to be run on each of the slurmd's in the allocation. As slurmd's report that the epilog ran successfully, the nodes are returned to the partition.

\subsection{Queued (batch) job initiation}

Figure 6 illustrates the initiation of a queued job in SLURM. The user invokes srun in batch mode by supplying the --batch option to srun. Once user options 
are processed, srun sends a batch job request to slurmctld that contains the input/output location for the job, current working directory, environment, requested number of nodes, etc. The slurmctld queues the request in its priority ordered queue.

Once the resources are available and the job has a high enough priority, slurmctld allocates the resources to the job and contacts the first node of the allocation requesting that the user "job" be started. In this case the job may either be another invocation of srun or a job script which may have multiple invocations of srun within it. The slurmd on the remote node responds to the run request, initiating the job thread, task thread, and user script. An srun executed from within the script detects that it has access to an allocation and initiates a job step on some or all of the nodes within the job.

Once the job step is complete, the srun in the job script notifies the slurmctld and terminates. The job script continues executing and may initiate further job steps. Once the job script completes, the task thread running the job script collects the exit status and sends a task exit message to the slurmctld. The slurmctld notes that the job is complete and requests that the job epilog be run on all nodes that were allocated. As the slurmd's respond with successful completion of the epilog, the nodes are returned to the partition.

\subsection{Allocate mode initiation}

In allocate mode, the user wishes to allocate a job and interactively run job steps under that allocation. The process of initiation in this mode is illustrated in figure 7 . The invoked srun sends an allocate request to slurmctld, which, if resources are available, responds with a list of nodes allocated, time limit, etc. The srun process spawns a shell on the user's terminal with access to the allocation, then waits for the shell to exit (at which time the job is considered complete).

An srun initiated within the allocate subshell recognizes that it is running under an allocation and therefore already within a job. Provided with no other arguments, srun started in this manner initiates a job step on all nodes within the current job. However, the user may select a subset of these nodes implicitly by using the srun --nodes option, or explicitly by specifying a relative nodelist ( --nodelist=[0-5]).

An srun executed from the subshell reads the environment and user options, then notify the controller that it is starting a job step under the current job. The slurmctld registers the job step and responds with a job credential. srun then initiates the job step using the same general method as described in the section on interactive job initiation.

When the user exits the allocate subshell, the original srun receives exit status, notifies slurmctld that the job is complete, and exits. The controller runs the epilog on each of the allocated nodes, returning nodes to the partition as they complete the epilog. 


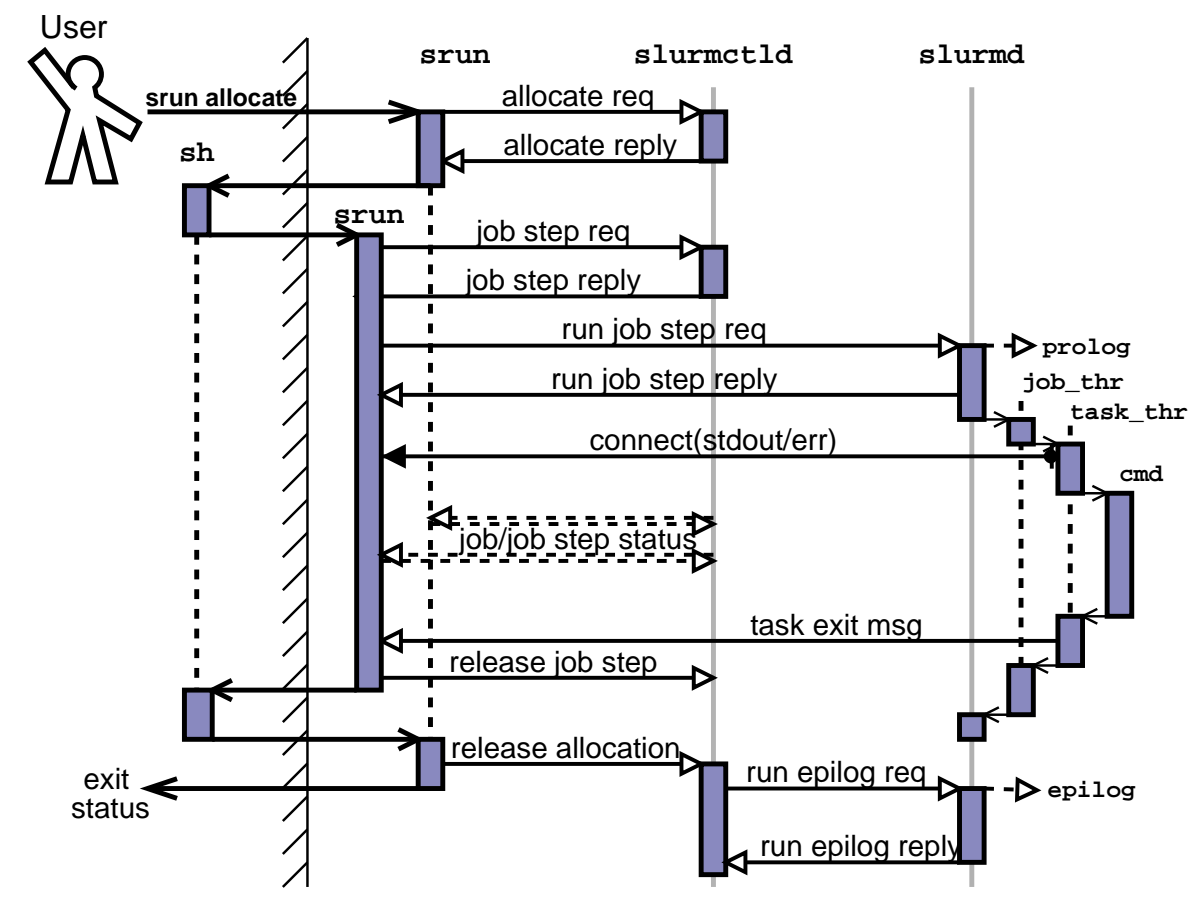

Figure 7: Job initiation in allocate mode. Resources are allocated and srun spawns a shell with access to the resources. When user runs an srun from within the shell, the a job step is initiated under the allocation.

\section{Infrastructure}

The state of slurmctld is written periodically to disk for fault tolerance. SLURM daemons are initiated via inittab using the respawn option to insure their continuous execution. If the control machine itself becomes inoperative, its functions can easily be moved in an automated fashion to another computer. In fact, the computer designated as alternative control machine can easily be relocated as the workload on the compute nodes changes.

The syslog tools are used for logging purposes and take advantage of the severity level parameter.

Direct use of the Elan interconnect is provided a version of MPI developed and supported by Quadrics. SLURM supports this version of MPI with no modifications. Support of MPICH is expected shortly.

SLURM supports the TotalView debugger[3]. This requires srun to not only maintain a list of nodes used by each job step, but also a list of process IDs on each node corresponding the application's tasks. 


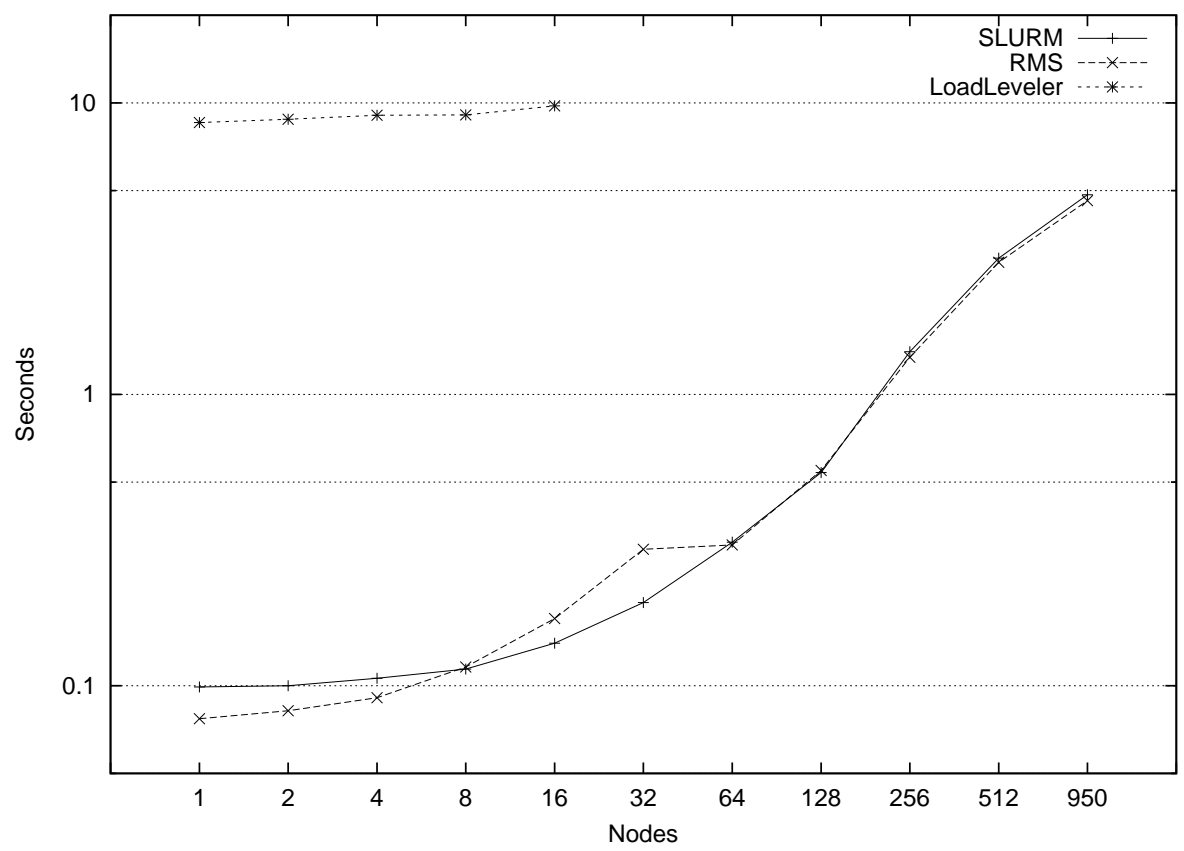

Figure 8: Time to execute /bin/hostname with various node counts

\section{Results}

We were able to perform some SLURM tests on a 1000 node cluster in November 2002. Some development was still underway at that time and tuning had not been performed. The results for executing the program /bin/hostname on two tasks per node and various node counts is show in figure 8. We found SLURM performance to be comparable to RMS for all job sizes and about 100 times faster than LoadLeveler at small job sizes. (While not shown on this chart, LoadLeveler reaches 1200 seconds to launch an 8000 task job on 500 nodes.)

\section{Future plans}

As of December 2002, some work remains before we feel ready to distributed SLURM for general use. Work needed at that time was primarily in configurability, MPICH support, TotalView support, scalability, fault-tolerance, job accounting, and testing. We expect SLURM to begin production use on LLNL Linux clusters in January 2003.

Looking ahead, we anticipate porting SLURM to the IBM Blue Gene[5] in 2003. Blue Gene has a different interconnect than any supported by SLURM. It also has a 3-D topography with restrictive allocation constraints. 


\section{Acknowledgements}

- Chris Dunlap for technical guidance

- Joey Ekstrom and Kevin Tew for their work developing the communications infrastructure and user tools

- Jim Garlick for his development of the Quadrics Elan interface and technical guidance

- Gregg Hommes, Bob Wood and Phil Eckert for their help designing the SLURM APIs

- David Jackson of Linux Networx for technical guidance

- Fabrizio Petrini of Los Alamos National Laboratory for his work to integrate SLURM with STORM communications

- Mark Seager and Greg Tomaschke for their support of this project

- Jay Windly of Linux Networx for his work on the security components 


\section{A Glossary}

Authd User authentication mechanism

DCE Distributed Computing Environment

DFS Distributed File System (part of DCE)

DPCS Distributed Production Control System, a meta-batch system and resource manager developed by LLNL

Globus Grid scheduling infrastructure

Kerberos Authentication mechanism

LoadLeveler IBM's parallel job management system

LLNL Lawrence Livermore National Laboratory

Munged User authentication mechanism developed by LLNL

NQS Network Queuing System (a batch system)

OSCAR Open Source Cluster Application Resource

RMS Quadrics' Resource Management System

TotalView Etnus' debugger 


\section{References}

[1] Eitan Frachtenberg, Fabrizio Petrini, et al. Storm: Lightning-fast resource management. In Proceedings of SuperComputing, 2002.

[2] Authd home page. http://www.theether.org/authd/.

[3] Etnus home page. http://www.etnus.com.

[4] Globus home page. http://www.globus.org.

[5] LLNL BlueGene/L home page. http://www.llnl.gov/asci/platforms/bluegenel.

[6] David Jackson, Quinn Snell, and Mark Clement. Core algorithms of the maui scheduler. In Dror Feitelson and Larry Rudolph, editors, Job Scheduling Stategies for Parallel Processing, volume LLCS 2221, pages 87-102. Springer Verlag, 2002.

[7] Moe Jette et al. Survey of batch/resource management-related system software. Technical report, LLNL, 2002.

[8] DPCS overview. http://www.llnl.gov/icc/lc/dpcs/dpcs_overview.html.

[9] Maui scheduler repository. http://mauischeduler.sourceforge.net/. 